Rev. Adm. Saúde - Vol. 18, № 70, jan. - mar. 2018

http://dx.doi.org/10.23973/ras.70.94

RELATO DE CASO

\title{
Mapeamento do processo de alta para pacientes de ambulatório cirúrgico baseado em contrarreferência de um hospital público de ensino de alta complexidade do município do São Paulo
}

Discharge process mapping of surgical outpatients based on counter-referral of a high complexity public teaching hospital in the city of São Paulo

\section{Jaqueline Oliveira Valdevino Nascimento', Ana Cláudia Becker²,} Rosangela Eiroz ${ }^{3}$, Paula Cristina Souto de Camargo ${ }^{4}$

1. Enfermeira. Aprimoranda do PROAHSA - Programa de Estudos Avançados em Administração Hospitalar e Sistemas de Saúde - HCFMUSP, São Paulo SP.

2. Gerontóloga. Aprimoranda do PROAHSA - Programa de Estudos Avançados em Administração Hospitalar e Sistemas de Saúde - HCFMUSP, São Paulo SP.

3. Administradora de Empresas. Administradora no Departamento Gastroenterologia HCFMUSP, São Paulo SP.

4. Especialista em Administração Hospitalar e de Sistemas. Coordenadora de Ensino do PROAHSA - Programa de Estudos Avançados em Administração Hospitalar e Sistemas de Saúde - HCFMUSP, São Paulo SP.

\section{RESUMO}

Introdução: Na gestão hospitalar, sistemas de referência e contrarreferência efetivos atuam como elementos essenciais para a integração das redes de saúde diante da complexidade dos serviços. Objetivo: Mapear o processo de alta de um hospital público de ensino de alta complexidade do município do São Paulo e criar protocolos de contrarreferência para pacientes atendidos nos ambulatórios do Serviço de Cirurgia do Esôfago e Fígado e Hipertensão Portal. Método: Estudo descritivo, com revisão bibliográfica e realização de Benchmarking, sendo desenvolvida uma proposta de melhoria para a área interessada. Resultado: Foi realizado um diagnóstico situacional do setor ambulatorial onde se notou uma falta de padronização em relação ao documento utilizado e elaborados relatórios de alta e protocolos para 
diagnósticos específicos de acordo com os protocolos terapêuticos dos grupos analisados. Conclusão: Faz-se necessário a criação de protocolos de contrarreferência a fim de diminuir a demanda e a superlotação dos hospitais de alta complexidade com casos que poderiam ser sanados na rede de saúde com atenção menos especializada, otimizando assim o sistema de saúde e respeitando seu caráter hierarquizado e de rede.

Palavras-chave: administração de serviços de saúde, assistência ambulatorial, administração hospitalar, gestão em saúde, hospitais públicos, hospitais de ensino.

\section{ABSTRACT}

Introduction: In hospital management, effective referral and counter-referral systems act as essential elements for the health networks integration in the face of service complexity. Objective: To map the discharge process of a high complexity public teaching hospital in the city of São Paulo and create counterreferral protocols for patients attending the Ambulatory Esophageal and Liver Surgery and Portal Hypertension Clinical Services. Method: A descriptive study, with a bibliographic review and Benchmarking, and an improvement proposal were developed for the area. Outcome: A situational diagnosis was performed in the outpatient clinic where a lack of document standardization was observed, then discharge reports and protocols for specific diagnoses were elaborated according to the therapeutic protocols of the analyzed groups. Conclusion: It is necessary to create counter-referral protocols in order to reduce the demand and overcrowding of highly complex hospitals with cases that could be treated in the health network with less specialized health services, thus optimizing the health system and respecting its hierarchical and network character.

Keywords: health services administration; ambulatory care; hospital administration; hospital administration; health management; hospitals, public; hospitals, teaching.

\section{INTRODUÇÃO}

Segundo Vecina (2011), o ambulatório é caracterizado como a modalidade de atuação a pacientes em regime de não internação ${ }^{(1)}$. Os parâmetros populacionais para fins de cálculo da demanda e planejamento de unidades ambulatoriais são voltados ao número de consultas médicas por habitante/ano. No Brasil, no setor público, estes índices oscilam entre 2 e 3 consultas por habitante/ano (Portaria 1.101/2002 do Ministério da Saúde) ${ }^{(2)}$. No setor privado esse número é de 5,36 consultas/habitante/ano, constatado em levantamento da Agência Nacional de Saúde Suplementar (ANS), $2004{ }^{(3)}$. 
No sistema de saúde, as diferentes modalidades de assistência são organizadas em três níveis de complexidade: Atenção Primária; Atenção Secundária; e Atenção Terciária. Nesse sentido, o Ministério da Saúde ${ }^{(4)}$ estabelece que um efetivo sistema de referência e contrarreferência é entendido como o mecanismo de encaminhamento mútuo de pacientes entre os diferentes níveis de complexidade dos serviços, sendo o principal elemento para a integração das redes de saúde. O atendimento integral à saúde, presente nos textos da Constituição Federal de 1988 e estabelecido como um dos princípios do SUS atua como um eixo prioritário de uma política de saúde, uma vez que representa a superação de obstáculos e a implantação de inovações no cotidiano dos serviços de saúde, nas relações entre os níveis de gestão do SUS e nas relações destes com a sociedade ${ }^{(5,6)}$.

Estudo realizado no Rio de Janeiro sobre o sistema de referência e contrarreferência constatou que, entre os fatores que dificultam o acesso dos pacientes e o bom funcionamento dos sistemas de referência e contrarreferência nas áreas pesquisadas, estão: limitada oferta de consultas e exames; inexistência ou precariedade da contrarreferência; má organização das atividades de regulação; baixa utilização de protocolos clínicos para encaminhamentos; precariedade em termos de sistemas de informação e comunicação; significativa influência política na gestão das unidades; grande diversidade na denominação das unidades de saúde e multiplicidade das grades de oferta de serviços ${ }^{(7,8)}$.

A ocupação de vagas nos ambulatórios por pacientes que não necessitam de um atendimento em hospital terciário ocasiona a falta de rotatividade necessária para que haja o enfrentamento da superlotação vivenciada pelos sistemas de saúde, gerando, consequentemente, um aumento ainda maior das filas de esperas para pacientes que necessitam realizar a primeira consulta no serviço, sobrecarregando, consequentemente, o sistema de saúde ${ }^{(9,10)}$.

Sendo demonstrado por um estudo realizado sobre gastos em saúde que os fatores que agem na demanda por serviços de saúde estão associados, principalmente: a necessidade sentida, fatores psicossociais, seguridade social, demografia, epidemiologia, utilização dos serviços, regulamentação e fatores culturais. E os agem na oferta da assistência à saúde são: progresso técnicomédico, difusão da inovação e multiplicação dos centros de assistência à saúde (11).

O aumento de gastos desnecessários com os atendimentos compromete a manutenção adequada do serviço em funcionamento, uma vez que, deixa de investir em melhorias na estrutura da instituição, e compromete, consequentemente, a possibilidade de criar ambientes melhores para os enfermos, buscar novas tecnologias de saúde, e investir na própria capacitação dos profissionais. E, tratando-se de uma instituição voltada para a assistência, o ensino e pesquisa, deve-se considerar que, o atendimento de casos que deveriam ocorrer em um nível de complexidade primário ou secundário, implica no prejuízo da aprendizagem teórica e prática de estudantes que estão em processo de formação dentro do instituto ${ }^{(12)}$. 
Nesse sentido, a pergunta que direciona este trabalho é: como ocorre o processo de alta para os pacientes dos ambulatórios? Desse modo, o objetivo deste trabalho consiste em analisar o processo de alta para pacientes dos Ambulatórios do Serviço de Cirurgia do Esôfago e Fígado e Hipertensão Portal, ligados à Divisão de Cirurgia do Aparelho Digestivo e Coloproctologia de um hospital público de ensino de alta complexidade do município do São Paulo.

O artigo é uma contribuição para a área de Administração Geral e Pública, dada a importância de um efetivo sistema de referência e contrarreferência entre os diferentes níveis de complexidade dos serviços. E diante deste cenário, tem-se que a falha nesse processo pode acarretar em diversos problemas para as instituições de saúde, sendo elas relacionadas, por exemplo: ao aumento dos gastos hospitalares; à falta de rotatividade dos pacientes; ao aumento da fila de espera hospitalar; e ao prejuízo em relação a aprendizagem dos estudantes dentro de um hospital escola.

\section{OBJETIVO}

Este projeto tem como objetivo mapear o processo de alta e criar protocolos de contrarreferência para pacientes dos Ambulatórios do Serviço de Cirurgia do Esôfago e Fígado e Hipertensão Portal, ligados à Divisão de Cirurgia do Aparelho Digestivo e Coloproctologia de um hospital público de ensino de alta complexidade do município do São Paulo.

\section{MÉTODOS}

Trata-se de um estudo descritivo, com revisão bibliográfica, que apresentou o período de um mês para a sua realização, tendo como base a metodologia de gestão de projetos, realizado de um hospital público de ensino de alta complexidade do município do São Paulo. Este hospital é referência para alta complexidade na atenção à saúde e, para isso, conta com um Departamento de Gastroenterologia que reúne as Disciplinas de Gastroenterologia e Hepatologia Clínica, Cirurgia do Aparelho Digestivo, Coloproctologia e Transplantes de Órgãos do Sistema Digestivo. A Divisão de Cirurgia do Aparelho Digestivo e Coloproctologia, em especial, divide-se em seis diferentes grupos: Serviço de Cirurgia Estômago, Duodeno e Intestino Delgado; Serviço de Cirurgia de Fígado e Hipertensão Portal; Serviço de Cirurgia Esôfago; Serviço de Cirurgia de Procotológica - Colon, Reto e Ânus; Serviço de Cirurgia de Vias Biliares e Pâncreas; e Unidade de Cirurgia Metabólica e Obesidade; que atendem em dezesseis consultórios diferentes.

Optou-se por selecionar dois dos seis grupos presentes na Divisão de Cirurgia do Aparelho Digestivo e Coloproctologia, para a realização do estudo, sendo eles: o Serviço de Cirurgia Esôfago e o Serviço de Cirurgia de Fígado e Hipertensão Portal. A escolha dos grupos baseou-se, principalmente, no engajamento dos integrantes da equipe em relação à proposta de projeto apresentada pelas responsáveis do projeto, visto que para a elaboração dos 
formulários de contrarreferência seria de suma importância à participação ativa das equipes para o fornecimento de dados necessários.

Para a coleta dos dados e compreensão a respeito da maneira como o processo de contrarreferência é realizado e, verificar a presença de padronização nas ações, foi elaborado um questionário com questões abertas. Os participantes foram contatados pessoalmente e optaram em participar ou não do estudo. Todos os membros de ambos os grupos selecionados foram abordados e aceitaram participar do estudo, contabilizando um total de dez participantes, sendo seis pertencentes ao Serviço de Cirurgia Esôfago e quatro do Serviço de Cirurgia de Fígado e Hipertensão Portal. As entrevistas foram realizadas nos consultórios dos ambulatórios e tiveram duração de 30 minutos aproximadamente, sendo as entrevistas norteadas pelas questões e pelos temas descritos no Quadro 1.

Quadro 1. Descritivo dos temas e questões norteadoras.

\begin{tabular}{|l|l|}
\hline Temas & Questões norteadoras \\
\hline Conceito de contrarreferência & O que é contrarreferência? \\
\hline Utilização na prática & $\begin{array}{l}\text { Como você realiza a contrarreferência } \\
\text { dos pacientes deste ambulatório? }\end{array}$ \\
\hline
\end{tabular}

Junto a esse processo também foram realizadas visitas observacionais para compreender a rotina do Ambulatório a fim de entender seu funcionamento, fluxos, atividades e profissionais envolvidos nas diferentes etapas de trabalho. Além das atividades junto à divisão, houve momentos em setores ligados ao ambulatório que contribuem para o seu funcionamento, como a Divisão de Arquivo Médico (DAM), Matrícula, Recepção e Faturamento. Durante esse período, a equipe do presente estudo pôde participar das reuniões semanais com os grupos da divisão, em que são discutidas as principais demandas e gargalos de cada grupo, a fim de buscar a melhoria contínua do grupo, e nortear os membros da equipe em relação às atividades com necessidades mais imediatas.

Neste estudo, foi realizado benchmarking com a Gastroenterologia Clínica do hospital apresentado, visto que se trata de um elemento importante para o andamento do estudo. Na visita realizada foi apresentado o processo de contrarreferência dos pacientes pertencentes à Gastroenterologia Clínica e abordado os casos específicos que justificam a transferência do paciente matriculado no hospital entre os diferentes grupos existentes, do Grupo de Cirurgia do Aparelho Digestivo para a Gastroenterologia Clínica, mediante necessidades específicas. E utilizaram-se ferramentas de gestão com o intuito de analisar as informações coletadas, auxiliar a mapear e diluir os riscos presentes durante sua realização, sendo elas: Análise SWOT e Matriz de Gestão de Risco (13). 


\section{RESULTADOS E DISCUSSÃO}

$\mathrm{Na}$ Divisão de Cirurgia do Aparelho Digestivo e Coloproctologia ao analisarmos os atendimentos ambulatoriais realizados no período de fevereiro/2017, em ambos os grupos selecionados para a realização do estudo, especificamente, constata-se que no Ambulatório do Serviço de Cirurgia do Esôfago, do total de 495 atendimentos ambulatoriais realizados: $81,2 \%$ eram retornos; $9,1 \%$ primeiras consultas; e $9,7 \%$ altas.

Já no Ambulatório do Serviço de Cirurgia de Fígado e Hipertensão Portal, do total de 374 atendimentos ambulatoriais realizados: $94,4 \%$ eram retornos; $5,6 \%$ primeiras consultas; e que não houve nenhuma alta. Sendo que tal frequência ocorreu de maneira uniforme nos anos anteriores, em ambos os grupos que fazem parte da Divisão de Cirurgia do Aparelho Digestivo e Coloproctologia, conforme representado nos gráficos a seguir.

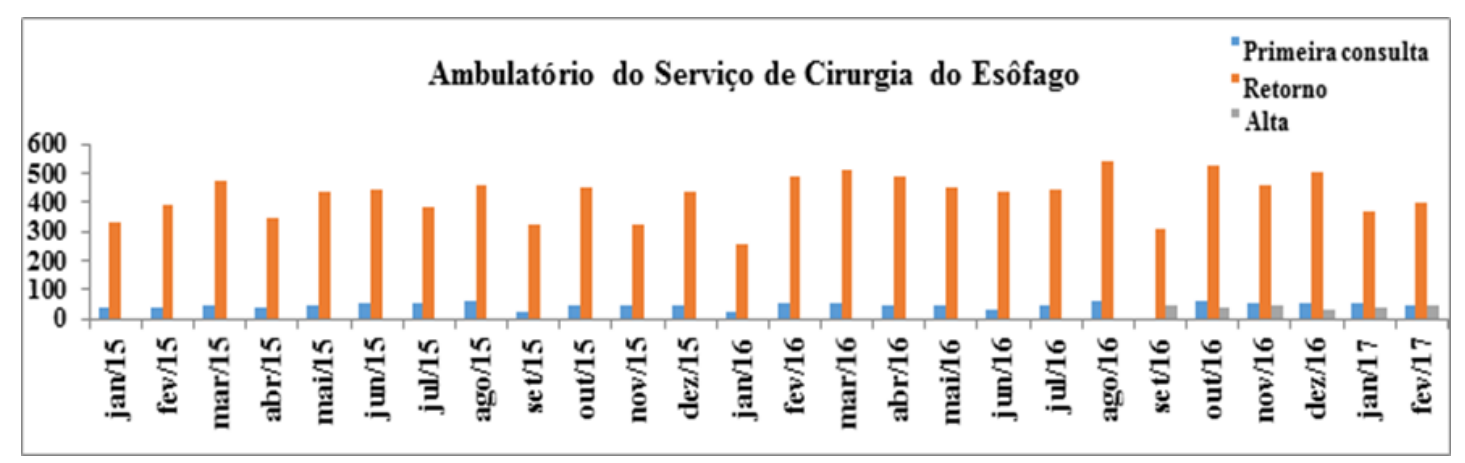

Gráfico 1: Rastreio das consultas ambulatoriais do grupo de cirurgia do Esôfago.

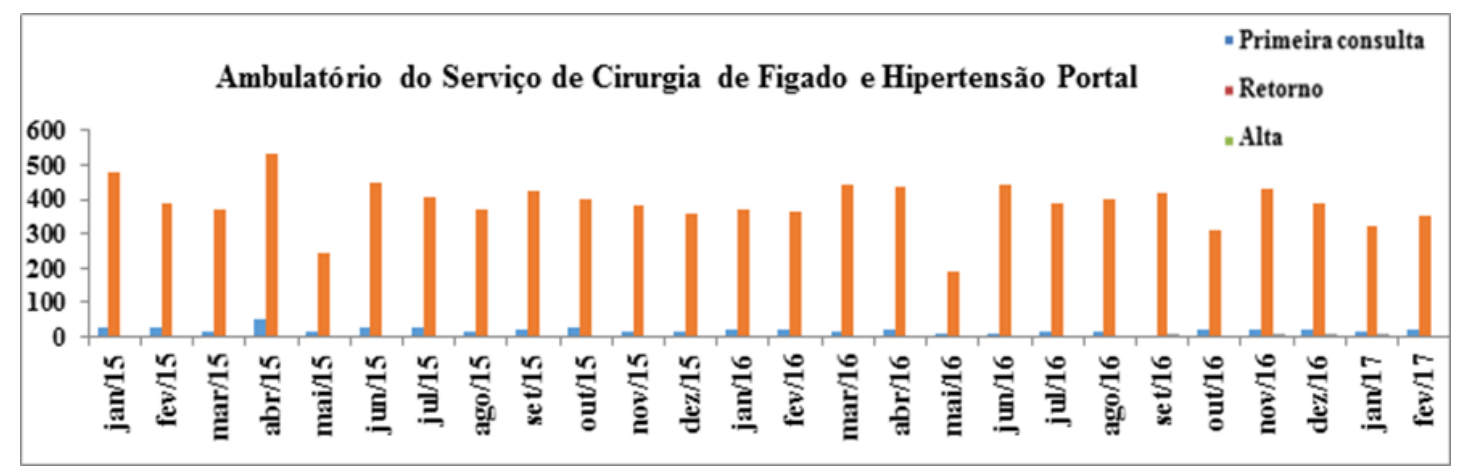

Gráfico 2: Rastreio das consultas ambulatoriais do grupo de cirurgia do Fígado e Hipertensão Portal.

Diante deste cenário e após o entendimento do perfil dos pacientes atendidos no ambulatório da gastrocirurgia, participação das reuniões de acompanhamento dos grupos com a consultoria, entrevistas realizadas com 
todos os membros de ambos e grupos, e reuniões específicas com cada grupo para entender as peculiaridades relacionadas com sua especialidade, elaborou-se diferentes documentos com o intuito de atender as necessidades apresentadas por cada grupo.

Os documentos gerais foram elaborados com o intuito de atender as necessidades de ambos os grupos contemplados pelo estudo. De forma geral, a equipe do estudo, desenvolveu: um protocolo de alta final; um protocolo de alta intermediário; um manual explicativo a respeito do preenchimento do protocolo de alta e um para alta intermediária; e um folheto explicativo para os pacientes, sendo utilizado como referencial o estudo de Salvador et al. (2009) que apresenta um modelo de relatório de alta para pacientes de fisioterapia de uma maneira clara e precisa ${ }^{(14)}$.

O Relatório de Alta Final (Quadro 2), é um documento elaborado para ser utilizado por ambos os grupos da Divisão de Cirurgia do Aparelho Digestivo e Coloproctologia. O profissional responsável pelo preenchimento do relatório, indica se a alta do paciente será para a rede de saúde ou se se trata de uma transferência para outra especialidade da própria instituição. Além disso, preenche os dados referentes ao Resumo Clínico do paciente, que contempla os seguintes campos:

- histórico do paciente: diagnóstico de acompanhamento pelo grupo (CID10); descrição e histórico do paciente junto ao grupo; cirurgias e intervenções realizadas durante o seguimento na instituição; e fatores de risco ou criticidades;

- $\quad$ situação atual do paciente;

- conduta para seguimento;

- recomendações para o paciente

Quadro 2. Relatório de Alta Final do Departamento de Cirurgia do Aparelho Digestivo.

\begin{tabular}{|l|l|}
\hline IDENTIFICAÇÃO DO HOSPITAL & $\begin{array}{l}\text { ETIQUETA DE IDENTIFICAÇÃO DO } \\
\text { PACIENTE }\end{array}$ \\
\hline $\begin{array}{l}\text { RELATÓRIO DE ALTA DO DEPARTAMENTO DE CIRURGIA DO APARELHO } \\
\text { DIGESTIVO }\end{array}$ & $\begin{array}{l}\text { Alta por transferência: } \\
\text { Gastroenterologia clínica: } \\
\text { Outra especialidade: }\end{array}$ \\
\hline $\begin{array}{l}\text { Ulta para a rede: } \\
\text { Especialidade: }\end{array}$ & \\
\hline RESUMO CLíNICO & \\
\hline Histórico do Paciente & \\
\hline Diagnóstico de acompanhamento pelo grupo & \\
\hline
\end{tabular}




\begin{tabular}{|l|l|}
\hline (CID-10): & \\
\hline $\begin{array}{l}\text { Descrição e histórico do paciente junto ao } \\
\text { grupo: }\end{array}$ & \\
\hline $\begin{array}{l}\text { Cirurgias e intervenções realizadas durante o } \\
\text { seguimento no HC: }\end{array}$ & \\
\hline Fatores de risco ou criticidades: & \\
\hline Situação atual do paciente & \\
\hline Conduta para seguimento \\
\hline Recomendações para o paciente \\
\hline
\end{tabular}

O Relatório de Aviso de Alta (Quadro 3), também elaborado para ser utilizado por ambos os grupos, atua como um aviso de alta. O intuito deste documento é possibilitar que o paciente, ao mesmo tempo em que está realizando seu tratamento e/ou acompanhamento na instituição hospitalar, consiga retornar a sua unidade de origem em busca de uma vaga, tendo em vista que ao término do período estipulado para sua permanência no hospital o paciente receba alta já sabendo em qual serviço terá seguimento. Possibilitando, desta forma, a redução dos conflitos entre médico-paciente no momento de alta, e consequentemente, uma maior segurança para o paciente a respeito da sua condição de saúde, além de ser um facilitador para o entendimento do profissional que dará sequência no atendimento deste paciente. Neste documento deve conter os dados referentes ao Resumo Clínico do paciente, que contempla os seguintes campos:

- histórico do paciente: diagnóstico de acompanhamento pelo grupo (CID10);

- $\quad$ situação atual do paciente;

- conduta para seguimento.

Quadro 3. Relatório de Aviso de Alta do Departamento de Cirurgia do Aparelho Digestivo. 
AVISO DE ALTA DO DEPARTAMENTO DE CIRURGIA DO APARELHO

DIGESTIVO

Encaminhamento para a rede

Unidade Básica de Saúde:

Especialidade:

RESUMO CLÍNICO

Histórico do Paciente

Diagnóstico de acompanhamento pelo

grupo (CID-10):

Situação atual do paciente

(cirurgias, intervenções e informações

relevantes do tratamento no

ambulatório):

Conduta para seguimento / preparação

para alta do HCFMUSP:

Médico responsável:

Data:

/

Os Manuais Explicativos de Alta Definitiva (Quadro 4) ou Relatório de Alta (Quadro 5), contém instruções para o preenchimento de ambos os documentos. Sua elaboração teve o intuito de facilitar a rotina dos profissionais que atuam no ambulatório, tendo em vista que devido à própria demanda da divisão, muitas vezes, o assistente não consegue realizar as orientações de como o processo de contrarreferência deve ser realizado no instituto, em tempo hábil para todos os residentes ou outros profissionais que apresentem dúvida. Assim, este manual ficará disponível dentro das salas de atendimento do setor, podendo ser consultados sempre que necessário.

Quadro 4. Instruções de Preenchimento do Relatório de Alta Final do Departamento de Cirurgia do Aparelho Digestivo.

\begin{tabular}{|c|c|}
\hline IDENTIFICAÇÃO DO HOSPITAL & LOGO GRUPO \\
\hline \multicolumn{2}{|c|}{ RELATÓRIO DE ALTA DO DEPARTAMENTO DE CIRURGIA DO APARELHO DIGESTIVO } \\
\hline \multicolumn{2}{|l|}{ IDENTIFICAÇÃO DO PACIENTE } \\
\hline \multicolumn{2}{|c|}{$\begin{array}{l}\text { Colar no local sinalizado no cabeçalho a etiqueta da instituição com os dados referentes } \\
\text { ao paciente. }\end{array}$} \\
\hline \multicolumn{2}{|c|}{ ALTA PARA A REDE } \\
\hline \multicolumn{2}{|c|}{$\begin{array}{l}\text { No local "ALTA PARA A REDE", identificar se o paciente será encaminhado à Unidade } \\
\text { Básica de Saúde para acompanhamento na Atenção Primária; No campo Especialidade, } \\
\text { identificar qual a especialidade que melhor atenderá as necessidades do paciente (Ex. } \\
\text { Gastroenterologia, Geriatria). }\end{array}$} \\
\hline
\end{tabular}


ALTA POR TRANSFERÊNCIA

- Se o paciente não se enquadrar no atendimento do Departamento de Cirurgia do Aparelho Digestivo, mas ainda assim necessitar de um atendimento na atenção terciária, pode-se fazer o pedido de Interconsulta na instituição, junto com o relatório de alta preenchido para o novo ambulatório de seguimento.

- Os encaminhamentos para a Gastroenterologia Clínica podem ser feitos mediante comunicação das duas equipes e também através da Interconsulta.

\section{RESUMO CLÍNICO}

- No resumo clínico estarão as informações primordiais para que o profissional que der continuidade ao tratamento compreenda o diagnóstico, as intervenções e necessidades do paciente, durante o período que fez tratamento junto ao grupo.

\section{DIAGNÓSTICO CIRÚRGICO}

- Informar o CID e diagnóstico inicial do paciente que motivou seu encaminhamento e tratamento junto ao grupo.

\section{DESCRIÇÃO E HISTÓRICO}

- Informações pertinentes sobre o histórico e antecedentes do paciente desde sua entrada no grupo, essenciais para a continuidade do tratamento por outro profissional.

\section{CIRURGIAS E INTERVENÇÕES}

- Descrever as cirurgias e intervenções realizadas no paciente, que estejam associadas ao seu diagnóstico e assistência no grupo.

\section{FATORES DE RISCO}

- Descrever os fatores de risco que interferem ou impactam no tratamento e continuidade dos cuidados do paciente, como comorbidades, hábitos e estilos de vida, entre outros.

\section{SITUAÇÃO ATUAL DO PACIENTE}

- Descrever o quadro de saúde atual do paciente, após seguimento e tratamento no ambulatório do grupo, que justifique seu encaminhamento para seguimento na Rede de Atenção em Saúde;

- Caso os objetivos propostos ou os resultados esperados não tenham sido alcançados, deverão ser mencionadas as razões para esse resultado, e para a determinação da alta;

\section{CONDUTA PARA SEGUIMENTO}

- Descrever a conduta ao médico da Rede de Saúde para que ele dê continuidade ao tratamento do paciente, de acordo com o que estava sendo feito no hospital;

- Apontar os objetivos que justifiquem o seguimento do paciente na Rede de Saúde em um nível de complexidade primário ou secundário, para a continuação dos cuidados; 
- Detalhar os exames necessários e a periodicidade para a realização dos mesmos.

\section{RECOMENDAÇÕES PARA O PACIENTE}

- Descrever ao paciente as condutas e hábitos para que seu tratamento e autocuidado se deem em sua completude, para uma melhora constante;

- Sensibilizar o paciente sobre seu papel e responsabilidade na continuidade dos cuidados, compartilhados com seu médico.

Quadro 5. Instruções de Preenchimento do Relatório de Aviso de Alta do Departamento de Cirurgia do Aparelho Digestivo.

IDENTIFICAÇÃO DO HOSPITAL

INSTRUÇÕES DE PREENCHIMENTO

AVISO DE ALTA DO DEPARTAMENTO DE CIRURGIA DO APARELHO DIGESTIVO

IDENTIFICAÇÃO DO PACIENTE

- Colar no local sinalizado no cabeçalho a etiqueta da instituição com os dados referentes ao paciente.

ENCAMINHAMENTO PARA A REDE

- No local "ENCAMINHAMENTO PARA A REDE", identificar se o paciente será encaminhado à Unidade Básica de Saúde para acompanhamento na Atenção Primária;

- No campo Especialidade, identificar qual a especialidade que melhor atenderá as necessidades do paciente (Ex. Gastroenterologia, Geriatria).

\section{RESUMO CLÍNICO}

- No resumo clínico estarão as informações primordiais para que o profissional que der início à continuidade do tratamento compreenda o diagnóstico, as intervenções e necessidades do paciente, para que possa acompanhar o caso em paralelo com o grupo.

\section{SITUAÇÃO ATUAL DO PACIENTE / CIRURGIAS E INTERVENÇÕES}

- Descrever o quadro de saúde atual do paciente, seu seguimento e tratamento no ambulatório do grupo, que justifique seu aviso de alta e encaminhamento para seguimento na Rede de Atenção em Saúde;

- Caso os objetivos propostos ou os resultados esperados não tenham sido alcançados, deverão ser mencionadas as razões para esse resultado, e para a determinação do aviso de alta;

- Descrever as cirurgias e intervenções realizadas no paciente, que estejam associadas ao seu diagnóstico e assistência no grupo.

\section{CONDUTA PARA SEGUIMENTO}

- Descrever a conduta ao médico da Rede de Saúde para que ele dê início à continuidade ao tratamento do paciente, de acordo com o que está sendo feito no hospital;

- Apontar os objetivos que justifiquem o seguimento do paciente na Rede de 


Saúde em um nível de complexidade primário ou secundário, para a
continuação dos cuidados;
Detalhar os exames necessários e a periodicidade para a realização dos
mesmos;
Detalhar o papel da atenção primária ou secundária em paralelo com o
hospital.

O Folheto Explicativo, foi elaborado para contemplar informações sobre o Sistema Único de Saúde, a instituição em que o estudo foi realizado e a sua complexidade. Este folheto deverá ser disponibilizado ao paciente no momento em que ele inicia seus atendimentos no hospital, assim desde o início, informase que, de acordo com sua complexidade e necessidade de tratamento, possui um período estipulado para a sua permanência no serviço. Proporcionando que o paciente se sinta parte responsável por seu tratamento, e desta forma, os itens selecionados para comporem o folheto foram:

- O que é o SUS e os níveis de complexidade;

- Complexidade da instituição analisada;

- Divisão de Cirurgia do Aparelho Digestivo e Coloproctologia;

- Marcação de consulta pela rede / Retorno pelo Sistema CROSS.

Posteriormente, foram elaborados documentos específicos para os grupos analisados com o intuito de atender as necessidades de todos os grupos de forma individual. A escolha dos diagnósticos contemplados em cada protocolo partiu da necessidade dos grupos trabalhados no projeto, de acordo com dados estatísticos a respeito de sua frequência em relação aos demais diagnósticos presentes no ambulatório. Os protocolos elaborados para os diagnósticos do Serviço de Cirurgia do Esôfago foram: estenose esofágica; megaesôfago; e doença do refluxo gastroesofágico, assim como exemplificado no quadro a seguir. Os documentos desenvolvidos para os demais diagnósticos. Já, os protocolos elaborados para os diagnósticos do Serviço de Cirurgia de Fígado e Hipertensão Portal (Quadro 7) foram: lesões hepáticas benignas; e hipertensão portal secundária à esquistossomose hepatoesplênica (EHE). Sendo que os documentos desenvolvidos para os demais diagnósticos contemplaram a mesma estrutura e informações principais.

Quadro 6. Protocolo de Consultas Segundo Tempo de Acompanhamento Ambulatório do Serviço de Cirurgia do Esôfago do Departamento de Cirurgia do Aparelho Digestivo.

IDENTIFICAÇÃO DO HOSPITAL

MEGAESFÔFAGO

Acompanhamento Pós-Cirúrgico 


\begin{tabular}{|c|c|}
\hline $\begin{array}{l}\text { TEMPO PÓS- } \\
\text { OPERATÓRIO }\end{array}$ & EXAMES / RECOMENDAÇÕES \\
\hline \multicolumn{2}{|l|}{1 MÊS } \\
\hline \multicolumn{2}{|l|}{3 MESES } \\
\hline 6 MESES & Folheto informativo \\
\hline 1 ANO & Endoscopia cromoscopia esofágica \\
\hline \multicolumn{2}{|l|}{2 ANOS } \\
\hline 3 ANOS & Endoscopia cromoscopia esofágica \\
\hline \multicolumn{2}{|l|}{4 ANOS } \\
\hline 5 ANOS & Endoscopia cromoscopia esofágica \\
\hline \multicolumn{2}{|l|}{6 ANOS } \\
\hline 7 ANOS & Endoscopia cromoscopia esofágica \\
\hline 8 ANOS & AVISO DE ALTA \\
\hline \multicolumn{2}{|l|}{9 ANOS } \\
\hline $\begin{array}{l}10 \text { ANOS } \\
\text { ALTA }\end{array}$ & Alta ambulatorial \\
\hline \multicolumn{2}{|c|}{ Relatórios de consulta } \\
\hline 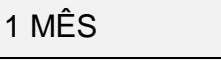 & Acompanhamento pós-cirúrgico \\
\hline 3 MESES & Acompanhamento pós-cirúrgico \\
\hline 6 MESES & $\begin{array}{l}\text { Acompanhamento pós-cirúrgico } \\
\text { Entrega do Folheto Informativo ao Paciente }\end{array}$ \\
\hline 1 ANO & $\begin{array}{l}\text { Acompanhamento pós-cirúrgico } \\
\text { Pedir Exames: Endoscopia com cromoscopia esofágica }\end{array}$ \\
\hline 2 ANOS & Checar Exames: Endoscopia com cromoscopia esofágica \\
\hline 3 ANOS & $\begin{array}{l}\text { Acompanhamento pós-cirúrgico } \\
\text { Pedir Exames: Endoscopia com cromoscopia esofágica }\end{array}$ \\
\hline 4 ANOS & Checar Exames: Endoscopia com cromoscopia esofágica \\
\hline 5 ANOS & $\begin{array}{l}\text { Acompanhamento pós-cirúrgico } \\
\text { Pedir Exames: Endoscopia com cromoscopia esofágica }\end{array}$ \\
\hline 6 ANOS & Checar Exames: Endoscopia com cromoscopia esofágica \\
\hline 7 ANOS & Pedir Exames: Endoscopia com cromoscopia esofágica \\
\hline 8 ANOS & $\begin{array}{l}\text { Checar Exames: Endoscopia com cromoscopia esofágica } \\
\text { Entregar Aviso de Alta preenchido ao paciente e orientar sobre rede de saúde }\end{array}$ \\
\hline 9 ANOS & $\begin{array}{l}\text { Perguntar sobre consulta na rede (UBS/Especialidade) } \\
\text { Pedir Exames: Endoscopia com cromoscopia esofágica }\end{array}$ \\
\hline $\begin{array}{l}10 \text { ANOS } \\
\text { ALTA }\end{array}$ & $\begin{array}{l}\text { Checar Exames: Endoscopia com cromoscopia esofágica } \\
\text { Entregar Relatório de Alta com exames recentes em anexo }\end{array}$ \\
\hline \multicolumn{2}{|l|}{ EXCEÇÕES } \\
\hline Esofagectomia & Manter o seguimento no $\mathrm{HC}$ \\
\hline
\end{tabular}


Quadro 7. Protocolo de Consultas Segundo Tempo de Acompanhamento Ambulatório do Serviço de Cirurgia de Fígado e Hipertensão Portal do Departamento de Cirurgia do Aparelho Digestivo.

\begin{tabular}{|c|c|}
\hline \multicolumn{2}{|c|}{ IDENTIFICAÇÃO DO HOSPITAL } \\
\hline \multicolumn{2}{|c|}{$\begin{array}{l}\text { HIPERTENSÃO PORTAL SECUNDÁRIA À ESQUISTOSSOMOSE } \\
\text { HEPATOESPLÊNICA (EHE) }\end{array}$} \\
\hline \multicolumn{2}{|c|}{ Acompanhamento Ambulatorial } \\
\hline $\begin{array}{l}\text { TEMPO PÓS } \\
\text { OPERATÓRIO }\end{array}$ & EXAMES /RECOMENDAÇÕES \\
\hline \multicolumn{2}{|l|}{6 MESES } \\
\hline 1 ANO & $\begin{array}{l}\text { USG ABDOME } \\
\text { Endoscopia } \\
\text { Folheto informativo }\end{array}$ \\
\hline 2 ANOS & $\begin{array}{l}\text { USG ABDOME } \\
\text { Endoscopia }\end{array}$ \\
\hline 3 ANOS & $\begin{array}{l}\text { USG ABDOME } \\
\text { Endoscopia } \\
\text { AVISO DE ALTA }\end{array}$ \\
\hline 4 ANOS & $\begin{array}{l}\text { USG ABDOME } \\
\text { Endoscopia }\end{array}$ \\
\hline $\begin{array}{l}5 \text { ANOS } \\
\text { ALTA }\end{array}$ & ALTA AMBULATORIAL \\
\hline \multicolumn{2}{|c|}{ Relatórios de consulta } \\
\hline 6 MESES & Acompanhamento ambulatorial \\
\hline 1 ANO & $\begin{array}{l}\text { Acompanhamento ambulatorial } \\
\text { Pedir exames: } \\
\text { Endoscopia e USG Abdome } \\
\text { Entrega do Folheto Informativo ao Paciente }\end{array}$ \\
\hline 2 ANOS & $\begin{array}{l}\text { Checar exames: Endoscopia e USG Abdome } \\
\text { Acompanhamento ambulatorial } \\
\text { Pedir exames: } \\
\text { Endoscopia e USG Abdome }\end{array}$ \\
\hline 3 ANOS & $\begin{array}{l}\text { Checar exames: Endoscopia e USG Abdome } \\
\text { Acompanhamento ambulatorial } \\
\text { Pedir exames: } \\
\text { Endoscopia e USG Abdome } \\
\text { Entregar Aviso de Alta preenchido ao paciente e orientar sobre rede } \\
\text { de saúde }\end{array}$ \\
\hline 4 ANOS & $\begin{array}{l}\text { Checar exames: Endoscopia e USG Abdome } \\
\text { Acompanhamento ambulatorial } \\
\text { Pedir exames: } \\
\text { Endoscopia e USG Abdome } \\
\text { Perguntar sobre consulta na rede (UBS/Especialidade) }\end{array}$ \\
\hline $\begin{array}{l}5 \text { ANOS } \\
\text { ALTA }\end{array}$ & $\begin{array}{l}\text { Checar exames: Endoscopia e USG Abdome } \\
\text { Entregar Relatório de Alta com exames recentes em anexo }\end{array}$ \\
\hline
\end{tabular}




\section{CONCLUSÃO}

A Divisão de Cirurgia do Aparelho Digestivo e Coloproctologia, após discussões realizadas entre os membros de suas equipes a respeito das características das consultas realizadas no ambulatório, verificou que muitos pacientes atendidos nos ambulatórios não apresentavam a complexidade necessária que configure a continuidade de seus atendimentos em um hospital terciário, todavia, os profissionais não realizavam a contrarreferência para as demais modalidades de assistência presentes na Rede de Saúde, de acordo com as necessidades dos pacientes.

Após a vivência dentro do cenário apresentado e participação nas reuniões com os diferentes grupos, foi possível realizar um diagnóstico situacional e apontar como principal problema a necessidade de elaboração do plano terapêutico para os pacientes ambulatoriais da Divisão de Cirurgia do Aparelho Digestivo e Coloproctologia, com o intuito de diminuir a demanda e a superlotação dos hospitais de alta complexidade com casos que poderiam ser sanados na rede de saúde com uma atenção menos especializada, otimizando assim o sistema de saúde e respeitando seu caráter hierarquizado e de rede. Visto que havia uma fragilidade no tocante à prática gerencial dos ambulatórios, o que, consequentemente, sobrecarregava os profissionais de funções administrativas e assistenciais.

A elaboração dos documentos com o intuito de atender as necessidades de ambos os grupos contemplados pelo estudo pode ser um recurso valioso para organizar e agilizar as ações dos profissionais nas várias dimensões de seu trabalho: assistência, educação e pesquisa, e no desempenho das funções gerenciais de forma efetiva e com qualidade.

Uma conclusão dos resultados da pesquisa é que, apesar dos profissionais validarem a importância desse processo, todo processo de mudança demanda tempo para a sua compreensão e incorporação na rotina, tornando, desta forma, as ações embasadas em uma prática crítica e reflexiva. Além disso, as dificuldades apresentadas pelos profissionais em reconhecer e utilizar ferramentas que auxiliem no processo gerencial e assistencial podem estar relacionadas a ausência de um fluxo de alta para a implementação de um efetivo sistema de contrarreferência para a rede de saúde e treinamento dos profissionais sobre esta prática.

O presente estudo não teve a pretensão de esgotar o assunto e tratou de um cenário específico da prática ambulatorial de um hospital de alta complexidade, da mesma forma que, espera-se a elaboração de pesquisas futuras com vistas a fornecer informações sobre o desenvolvimento de outros documentos ou utilização de ferramentas gerenciais específicas que possam ser utilizadas nos diversos âmbitos da prática profissional.

Por fim, atualmente, estima-se que os profissionais de saúde, continuamente, ampliem e desenvolvam seus conhecimentos sobre ferramentas gerenciais e competências que os apoiem nas tomadas de decisão com o intuito de promover o cuidado de qualidade. Sendo que para esse processo torna-se 
fundamental a capacidade de comunicação, a organização, o planejamento e a boa gestão de pessoas.

\section{REFERÊNCIAS}

1. VECINA, G. N.; MALIK, A. M. Gestão em Saúde. Rio de Janeiro: Editora Guanabara Koogan. 2ª Ed. 2016.

2. MINISTRO DE ESTADO DA SAÚDE. Portaria nํ1101/GM. Parâmetros Assistenciais do SUS. 2002. Disponível em:

http://www.betim.mg.gov.br/ARQUIVOS ANEXO/Portaria 1001;;20070606. pdf.

3. AGÊNCIA NACIONAL DE SAÚDE SUPLEMENTAR (Brasil). Rede assistencial e garantia de acesso na saúde suplementar. Rio de Janeiro: ANS. 2015. Disponível em: http://www.ans.gov.br/images/stories/Materiais para pesquisa/Materiais po $\underline{r \text { assunto/rede completo digital.pdf. }}$

4. BRASIL. Ministério da Saúde. Secretaria de Assistência à Saúde. Coordenação de Saúde da Comunidade. Saúde da Família: uma estratégia para a reorientação do modelo assistencial. Brasília. 1997.

5. CONSTITUIÇÃO DA REPÚBLICA FEDERATIVA DO BRASIL DE 1988. Disponível em:

http://www.planalto.gov.br/ccivil 03/constituicao/constituicao.htm

6. PINHEIRO, R. Integralidade em Saúde. In: Dicionário da Educação Profissional em Saúde. Disponível em:

http://www.epsjv.fiocruz.br/dicionario/verbetes/intsau.html.

7. SERRA, C. G.; RODRIGUES, P. H. A. Avaliação da referência e contrarreferência no Programa Saúde da Família na Região Metropolitana do Rio de Janeiro. Ciênc. saúde coletiva. 2010; 15(3): 3579- 3586.

Disponível em:

http://www.scielo.br/scielo.php?script=sci_arttext\&pid=S141381232010000900033.

8. SANTOS, M. G. Sistema de Referência - Contrarreferência em saúde em São Sebastião da Vitória, Distrito de São João Del Rei - MG: O papel da rede na atenção básica. Minas Gerais. 2015. Disponível em:

https://www.nescon.medicina.ufmg.br/biblioteca/imagem/Sistema de refere ncia contrarreferencia.pdf.

9. PROTASIO, A. P. L.; SILVA, P. B.; LIMA, E. C.; GOMES, L. B.; MACHADO, L. S.; VALENÇA, A.M.G. Avaliação do sistema de referência e contrarreferência do estado da Paraíba segundo os profissionais da Atenção Básica no contexto do $1^{\circ}$ ciclo de Avaliação Externa do PMAQ-AB. Rio de janeiro. 2014; 38: 209-220. Disponível em: 
https://www.nescon.medicina.ufmg.br/biblioteca/imagem/Sistema de refere ncia contrarreferencia.pdf.

10. ALVES, M. L. F.; GUEDES, H. M.; MARTINS, J. C. A.; CHIANCA, T. C. M. Rede de referência e contrarreferência para o atendimento de urgências em um município do interior de Minas Gerais - Brasil. Rev. Med. Minas Gerais. 2015; 25(4): 469-475. Disponível em: file:///C:/Users/G.R.J.J/Downloads/v25n4a02\%20.pdf.

11.BOAS, A. K. M. V. Desafios e estratégias para a gestão de hospitais universitários contemplados na literatura dos últimos 20 anos. Lavras - MG. 2015. Disponível em: http://www.prpg.ufla.br/admpublica/wpcontent/uploads/2015/12/DISSERTACAO Desafios-e-estrat\%C3\%A9giaspara-a-gest\%C3\%A3o-de-hospitais-universit\%C3\%A1rios-contempladosna-literatura-dos-\%C3\%BAltimos-20Anos.pdf.

12.ZUCCHI, Paola; DEL NERO, Carlos and MALIK, Ana María. Gastos em saúde: os fatores que agem na demanda e na oferta dos serviços de saúde. Saude soc. 2000. Vol.9, n.1-2, pp.127-150. Diponível em: http://www.scielo.br/scielo.php?script=sci arttext\&pid=S0104$\underline{12902000000100010 .}$.

13. Empreendedorismo para empreendedores | Endeavor Brasil. Disponível em: https://endeavor.org.br/.

14.SALVADOR, S., BETTENCOURT, C. ALVES, S. Modelo de relatório de alta de Fisioterapia: Validação de conteúdo. Salutis Scientia - Revista de Ciências da Saúde da ESSCVP. Vol.1. 2009.

Recebido: 19 de fevereiro de 2018. Publicado: xx

Correspondência: Jaqueline Oliveira Valdevino Nascimento. E-mail: jake_valdevino@hotmail.com

Conflito de Interesses: os autores declararam não haver conflito de interesses. 